\title{
PLANETARY NEBULAE IN THE MAgELLANiC CLOUDS
}

\author{
BENGT E. WeSTERLUND \\ (Steward Observatory, University of Arizona, Tucson, Ariz., U.S.A.)
}

\begin{abstract}
A BST RACT
The available photometric and spectroscopic data on the planetary nebulae in the Magellanic Clouds are discussed. Recent results regarding the distribution of the planetary nebulae in the Clouds and the kinematics of the Clouds are presented. Masses are determined for selected planetary nebulae in the Clouds, and the question about the most likely average mass is considered. The evolutionary tracks of the nuclei for two values of the average mass are compared with the actual positions in the HR diagram of the nuclei of the planetary nebulae in the Clouds.
\end{abstract}

\section{Introduction}

Planetary nebulae have been identified only in three external galaxies, M 31 and the Magellanic Clouds. Baade (1955) noted five possible planetary nebulae in M 31 . Recently, Miss Swope (1963) measured their mean photographic magnitude as 22.3, which, with an uncorrected distance modulus of $24 \cdot 8$, gives an absolute blue magnitude of $-2 \cdot 5$. Their large distances have so far limited further investigation.

In the Magellanic Clouds the surveys for planetary nebulae have been uniform and may be considered reasonably complete to an apparent blue magnitude of about 17.5-18. The most likely distance moduli of the Clouds are: $m-M=18.7$ for the Large Cloud (LMC), and $m-M=19$ for the Small Cloud (SMC). The limiting absolute blue magnitude of the surveys is then in the range -0.7 to -1.5 .

All the true planetary nebulae identified so far in the Magellanic Clouds are stellar. With presently available telescopes it should be possible to detect objects in the Clouds with radii exceeding $0 \cdot 3$ parsec. From the currently accepted theory for the evolution of planetary nebulae it follows that the absolute magnitude of objects that have expanded to this size should be about $M_{B}=+2$.

The planetary nebulae in the SMC belong to the 'Central System' and are found within 3.5 of the optical centre of the galaxy. In the LMC they belong to the 'Disk System', which has a radius of about $7^{\circ}$ (Westerlund, 1965, 1968). In the latter, the system is definitely flat and contains mostly 'Old Population-I' objects. In the SMC the structure is less clear, and it will be discussed below. 


\section{The Detection of Planetary Nebulae in the Magellanic Clouds}

\section{A. THE SMALL MAGELLANIC CLOUD}

Emission objects were identified in the SMC by Miss Cannon (1933). No object appears to have been classified as a planetary nebula until 1955, when Lindsay (1955) suggested that 17 objects in the SMC belonged to that class. The following year Koelbloed (1956) identified 16 objects as planetary nebulae. Henize's catalogue of emission nebulae (1956) contains a large number of unresolved objects; none is, however, identified as a planetary nebula.

It is of interest to consider Lindsay's criteria for the identification of planetary nebulae in the Clouds. They are: (1) The object should not be associated with nebulosity; (2) it should be generally stellar in appearance, and (3) show little or no continuum spectrum; (4) it should show typical discrete nebular lines, and (5) have positive color index.

Lindsay expanded his catalogue in 1956, listing 20 planetary nebulae and 9 possible planetary nebulae (Lindsay, 1956). In 1961 he published a new catalogue of emissionline stars and planetary nebulae in the SMC, listing 30 planetary nebulae (P) and 20 probable planetary nebulae (P?) among the 593 'point-source' emission-line objects (Lindsay, 1961). The objects in his classes $\mathrm{P}$ and $\mathrm{P}$ ? have photographic absolute magnitudes ranging between $-4 \cdot 7$ and $-0 \cdot 7$. The distribution of the absolute magnitudes differs significantly from that obtained for planetary nebulae in our Galaxy, as also noted by Svestka (1962). It is important both for the study of planetary nebulae and for studies of the nature of the Magellanic Clouds to establish whether the highluminosity objects are indeed normal planetary nebulae. The resolution of Lindsay's plates is about $10^{\prime \prime}$, or close to 3 parsec at the distance of the SMC. It is therefore possible that many of his objects are small diffuse nebulae. This has been confirmed by Henize and Westerlund (1963); of 14 objects brighter than $M_{\mathrm{pg}}=-3.0,11$ are clearly resolved on large-scale plates and their estimated masses range from 2 to 33 solar masses. It is therefore unlikely that these objects can be considered planetary nebulae. The unresolved objects are probably true planetary nebulae, and $M_{\mathrm{pg}}=-3 \cdot 0$ is the upper limit to the photographic absolute magnitude of this class, in reasonable agreement with the data for the galactic planetary nebulae.

\section{B. THE LARGE MAGELLANIC CLOUD}

Surveys for planetary nebulae in the LMC have been carried out by Westerlund and Rodgers (1959), Lindsay and Mullan (1963), and Westerlund and Smith (1964). Henize (1956) listed 97 unresolved $\mathrm{H} \alpha$-emission nebulae; of these Lindsay and Mullan's catalogue includes 63 .

There are 40 objects in common to the Westerlund-Smith (W-S) and the Lindsay- 
Mullan (L-M) catalogues. The former contains 2 objects not included in the latter. There are 25 objects in the L-M Table 1 not listed in the W-S catalogue, and an additional 44 possible planetary nebulae in the L-M Table 2 ; they are all faint, uncertain objects and will not be considered here. We have searched our plates for the 25 additional objects in the L-M Table 1 and we conclude: 2 objects appear as planetary nebulae on our blue spectral plates (they were earlier considered as plate flaws); 5 fall outside the field searched by us; 3 are definitely stars, 5 are resolved emission nebulae, and 10 objects cannot be seen at all on our plates.

At present we may consider that about 30 true planetary nebulae have been identified in the SMC and about 45 in the LMC to the limiting magnitude given above. All these objects belong to the most luminous group of planetary nebulae, and it may be assumed that their present phase of evolution occupies about $1 / 10$ of the lifetime of the nebulae. On this assumption, we find a total of approximately 300 planetary nebulae in the SMC and of 450 in the LMC. This may be compared with the estimated number of $4.8 \times 10^{4}$ planetary nebulae in our Galaxy (O'Dell, 1962). The masses of the three galaxies are about $1.5 \times 10^{9}$ solar masses for the SMC, $6 \times 10^{9}$ for the LMC, and $10^{11}$ for the Galaxy. The number of planetary nebulae per unit mass is in reasonable agreement between the SMC and our Galaxy, whereas there is a lack of planetaries in the LMC.

\section{The Distribution and Motions of the Planetary Nebulae in the Clouds}

\section{A. THE SMALL MAGELLANIC CLOUD}

Lindsay (1961) concluded that the planetary nebulae showed a preference for the Southern and Southwestern parts of the SMC, and that they were almost absent East of NGC $346\left(R . A . \sim 1^{\mathrm{h}}\right)$. Most of the objects rejected by Henize and Westerlund (1963) as non-planetary are in the core of the SMC. The remaining objects possibly show a slight concentration to the preceding edge of the Bar, most of the fainter objects (of less certain classification) are fairly well scattered in the outer parts (Figure 1).

Hindman (1967) has recently suggested that the SMC is a slightly flattened system, seen practically edge-on. This makes it important to re-analyze the distribution of the planetary nebulae. In Hindman's model the radio centre of rotation is at $01^{\mathrm{h}} 03^{\mathrm{m}}-$ $72^{\circ} 45^{\prime}$ (1975) with the major axis in position angle $55^{\circ}$. We recall that the centre of the old population, represented by the red stars found on long-exposure infrared plates, appears to be near NGC $419\left(01^{\mathrm{h}} 08^{\mathrm{m}}-73^{\circ} 10^{\prime}\right)$, whereas the 'optical centre' (of bright blue stars) is near $00^{\mathrm{h}} 48^{\mathrm{m}}-73^{\circ} .3$. If we accept Hindman's model we find that only 8 planetary nebulae fall within 500 parsec $\left(0^{\circ} .5\right)$ of the equatorial plane of the SMC; they would then hardly belong to the disk population.

The line (plane) of symmetry of the planetary nebulae goes through the optical 


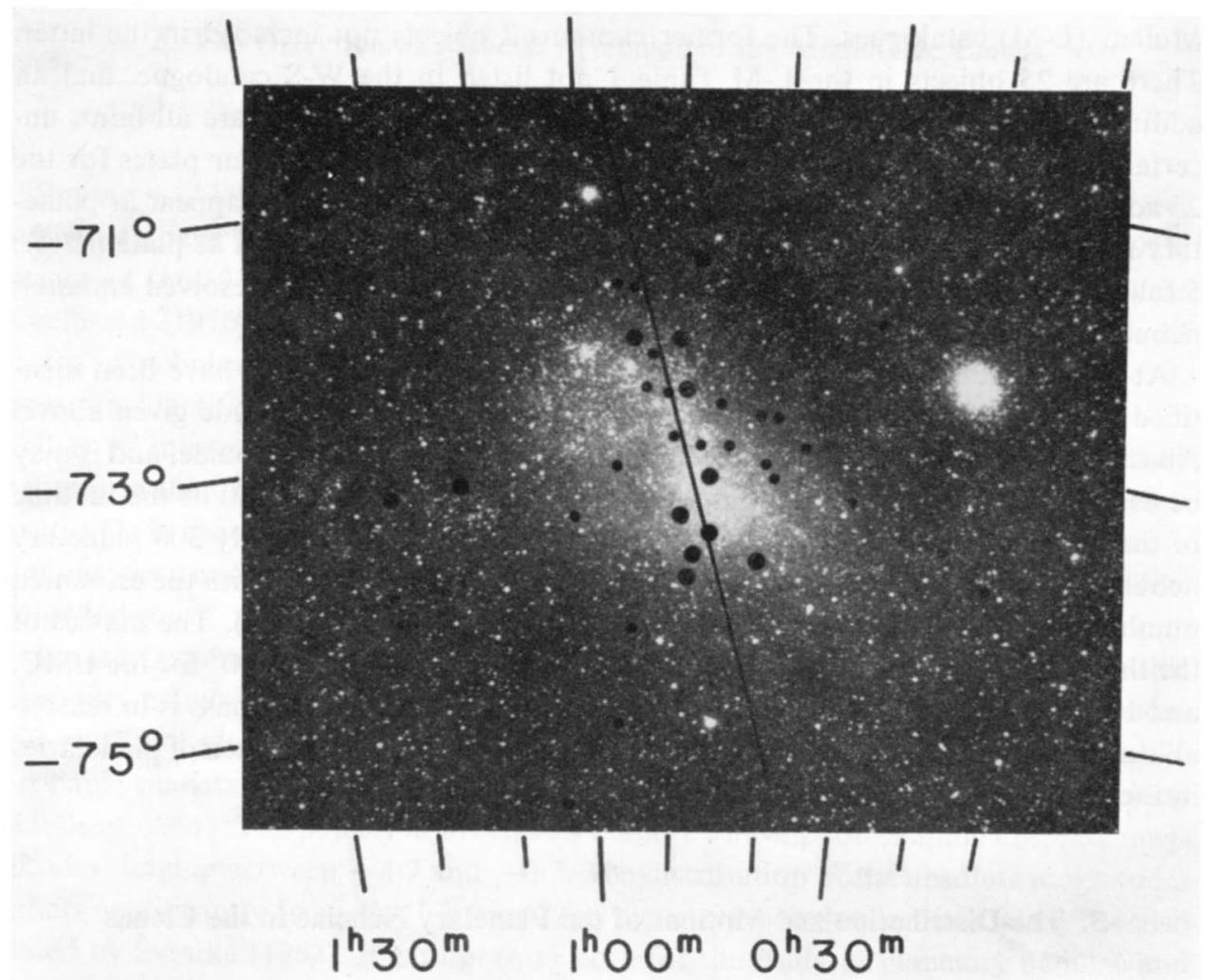

FIG. 1. The distribution of the planetary nebulae in the Small Magellanic Cloud. Large dots represent objects confirmed to be unresolved by Henize and Westerlund; small dots represent the objects in Lindsay's catalogue which have not yet been checked. The line of symmetry for the plotted objects is drawn.

centre of the SMC with a position angle of $16^{\circ} .25$ planetary nebulae, or $68 \%$, are within 500 parsec of this plane. Provided that the SMC is really an edge-on galaxy, we are inclined to consider this plane the true equatorial one, as it is defined with the aid of objects belonging to the old disk population in our Galaxy. This group is less likely to have been exposed to recent disturbances of the type that makes the present distribution of $\mathrm{HI}$ in the SMC so confusing.

Webster (1966) has determined velocities for 7 planetary nebulae in the SMC. All the velocities are smaller than the mean velocity of the SMC from Population-I objects (about $166 \mathrm{~km} / \mathrm{sec}$ ). They form two groups, one, consisting of 4 objects, with a mean velocity of $151 \mathrm{~km} / \mathrm{sec}$, and the second with a mean velocity of $119 \mathrm{~km} / \mathrm{sec}$. There is no connection between velocity and position in the SMC, and, consequently, no indication of any rotation. The different sub-systems in the SMC have obviously quite different characteristics in every way. 


\section{B. THE LARGE MAGELLANIC CLOUD}

The surface distribution of the planetary nebulae in the LMC appears circular with the centroid at $05^{\mathrm{h}} 22^{\mathrm{m}}-69^{\circ} .5$ (Westerlund and Smith, 1964). 50\% of the planetaries fall within $2 \mathrm{kpc}$ of this centre. Table IV in the W-S paper gives the centre of the various sub-systems; the so-called radio centre of rotation has recently been redetermined to $05^{\mathrm{h}} 20^{\mathrm{m}}-69^{\circ} .0$ (McGee and Milton, 1966), but this does not affect the discussion.

Webster (1966) has determined radial velocities for 13 planetary nebulae in the LMC and combined her data with Feast's (1964) measurements of 6 additional objects for a discussion of the kinematics of the LMC. The corrections for solar motion and galactic rotation as well as the systematic radial velocity of the LMC, the position angle of the maximum velocity gradient, and the inclination of the LMC to the plane of the sky were chosen to conform with previous analyses (Feast et al., 1961).

Rotation curves were derived about two centres: (I) the centre of symmetry of the extreme Population-I curve $\left(05^{\mathrm{h}} 20^{\mathrm{m}}-68^{\circ} .8\right)$, and (II) the centroid of the planetary nebulae sub-system $\left(05^{\mathrm{h}} 22^{\mathrm{m}}-69^{\circ} .5\right)$. Webster concludes that it is immediately obvious that the planetary rotational velocities deviate from those of the Extreme Population I near the centre of rotation of the latter population (Webster, 1965, 1966). They are more symmetrical around the centroid (II), which is close to the true mass centre of the LMC. Since the planetary nebulae may be expected to be less affected by gas streaming than the Extreme Population I, they are likely to give more reliable information about the gravitational field of the LMC. It is therefore suggested that the centre of rotation of the LMC coincides with the true centre of mass of the system, within the Bar. (See, however, Feast, 1964, and Feast's paper in this volume, p. 34.) No further analysis of the rotation curve is possible because of the obvious deviations from symmetry (due to the Bar) and possibly also from coplanarity (cf. McGee and Milton, 1966).

Webster suggests that three of the planetary nebulae, P 2, P 24 and P 33 (Westerlund and Smith, 1964), may move in very eccentric orbits. If these three objects are excluded, the dispersion of the remainder of the planetaries from the curve around centre (II) is $8.2 \mathrm{~km} / \mathrm{sec}$. This should be compared with the value of $9.6 \mathrm{~km} / \mathrm{sec}$ for the blue supergiants when the runaway stars are excluded. We note also that $\mathrm{McGee}$ and Milton (1966) find that in most cases the differences between the radial velocities of the planetary nebulae and the $\mathrm{H}$ I in their directions are quite large.

\section{Photometry of the Planetary Nebulae in the Clouds}

\section{A. NARROW-BAND PHOTOELECTRIC PHOTOMETRY}

Webster (1966) has determined the fluxes in the $\mathrm{N}_{1}, \mathrm{H} \beta, \mathrm{H} \gamma$, and [O $\left.\mathrm{II}\right] \lambda 3727$ lines, as well as the monochromatic magnitudes at $\lambda \lambda 5300,4200$ and 3500 for 10 planetary 
nebulae in the SMC and for 18 in the LMC. Her results are fundamental for the discussion in the following, and I am grateful for her permission to use them in advance of publication.

\section{B. HETEROCHROMATIC MAGNITUDES}

The photographic photometry of the planetary nebulae in the LMC (Westerlund and Smith, 1964) appears to be sufficiently accurate for an attempt to be made to interpret the red (R) and blue (B) magnitudes astrophysically. Comparison with Webster's data gives

$$
-\log F(\mathrm{H} \beta)=12 \cdot 33+0 \cdot 3\left(R_{\mathrm{n}}-14\right) .
$$

The subscript $n$ refers to the total nebula, an $s$ will refer to the central star, only. The scatter is less than $\pm 0 \cdot 1$ in $R_{\mathrm{n}}$.

In deriving relation (1) we have excluded 4 objects: P 7, P 9, P 17, and P 18 in the W-S catalogue. Two of these, P 7 and P 9, are known to have strong [N $\mathrm{NI}_{\text {II }}$ lines: this will affect the $R$ magnitude appreciably.

$B_{\mathrm{n}}$ is related to $m_{4200}: m_{4200}=B_{\mathrm{n}}+1 \cdot 03$.

The dispersion is here \pm 0.3 (16 objects), which clearly shows that other factors severely influence $B_{\mathrm{n}}$, notably the emission lines $\mathrm{N}_{1}, \mathrm{~N}_{2}, \mathrm{H} \beta$, and $\mathrm{H} \gamma$. We find

where

$$
-\log F(\Sigma)=11 \cdot 5+0 \cdot 3\left(B_{\mathrm{n}}-16 \cdot 4\right)
$$

$$
\begin{aligned}
F(\Sigma) & =F\left(\mathrm{~N}_{1}\right)+F\left(\mathrm{~N}_{2}\right)+F(\mathrm{H} \beta)+F(\mathrm{H} \gamma) \\
& \sim \frac{4}{3} F\left(\mathrm{~N}_{1}\right)+\frac{3}{2} F(\mathrm{H} \beta) .
\end{aligned}
$$

Webster's data show that for the LMC planetary nebulae we may write as a good approximation

$$
\log F\left(\mathrm{~N}_{1}\right) \sim \log F(\Sigma)-0 \cdot 20 .
$$

From (1), (3) and (4) we conclude that

$$
B_{\mathrm{n}}-R_{\mathrm{n}} \sim \log F(\mathrm{H} \beta) / F\left(\mathrm{~N}_{1}\right) .
$$

$B_{\mathrm{n}}-R_{\mathrm{n}}$ is thus a measure of the excitation of the nebulae; low colour indicates high excitation, and high (red) colour, low excitation. $R_{\mathrm{n}}$ is a measure of the flux in $\mathrm{H} \beta$. The excellent relation between $R_{\mathrm{n}}$ and $F(\mathrm{H} \beta)$ indicates that the $F(\mathrm{H} \alpha) / F(\mathrm{H} \beta)$ ratio is constant for the LMC objects.

\section{THE EXCITATION, HYDROGEN FLUX DIAGRAMS}

In her thesis Webster analyzes the $\log F\left(\mathrm{~N}_{1}\right) / F(\mathrm{H} \beta)$ vs. $\log F(\mathrm{H} \beta)$ diagram for the planetary nebulae in the two Clouds. She concludes that all the objects have $\log F(\mathrm{H} \beta)$ 
fainter than $-12 \cdot 3$; this defines an upper limit to the total emission of any planetary nebula. This upper limit may be expressed as a function of the excitation; there is a well-defined upper envelope on the high- as well as on the low-excitation side of $\log F\left(\mathrm{~N}_{1}\right) / F(\mathrm{H} \beta)=0 \cdot 74$; the slopes are

$$
\log F(\mathrm{H} \beta) \sim \begin{cases}-0.5 \log F\left(\mathrm{~N}_{1}\right) / F(\mathrm{H} \beta) & \text { high-excitation side } \\ +0.5 \log F\left(\mathrm{~N}_{1}\right) / F(\mathrm{H} \beta) & \text { low-excitation side. }\end{cases}
$$

However, it appears likely that at an $F\left(\mathrm{~N}_{1}\right) / F(\mathrm{H} \beta)$ value of about 10 the $\mathrm{H} \beta$ flux decreases rapidly without any obvious change in the ratio. The faintest object with a known $\mathrm{H} \beta$ flux, $\mathrm{P} 17$, falls about 0.6 in $\log F(\mathrm{H} \beta)$ below the envelope, yet its excitation ratio is close to 10 .

A certain confusion appears in the diagram as a mixture of high-excitation and low-excitation objects is found at about $F\left(\mathrm{~N}_{1}\right) / F(\mathrm{H} \beta)=10$ and about $0 \cdot 2$ below the high-excitation envelope. A comparison with the $\mathrm{H}-\mathrm{R}$ diagram for the nuclei (Figure 3 ) shows that 6 of the 8 objects in this group have low-temperature nuclei; the low $\mathrm{H} \beta$ flux is caused by the low temperature and luminosity of the star in an optically thick nebula whereas [OHI] is less sensitive to this.

According to our conclusions above, an $R_{\mathrm{n}},\left(B_{\mathrm{n}}-R_{\mathrm{n}}\right)$ diagram should give at least an approximation of a flux, excitation diagram. It is given for the LMC objects in Figure 2. It appears possible here also to define high- and low-excitation envelopes. We note that near the latter are the two objects, P 7 and P 9, with known strong [N II]

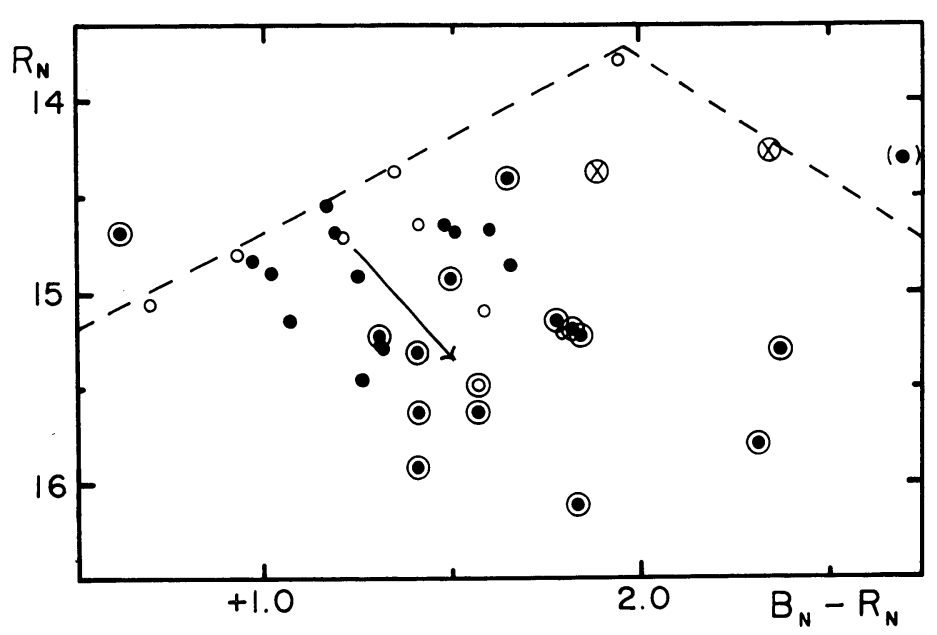

Fig. 2. The $R_{\mathrm{n}},\left(B_{\mathrm{n}}-R_{\mathrm{n}}\right)$ diagram for the planetary nebulae in the Large Magellanic Cloud. Circles represent planetary nebulae with observed He II 4686; crosses objects with known strong [ $\mathrm{N}_{\mathrm{II}}$ ] 6584. Large circles are drawn to identify objects situated to the West of $5^{h} 20^{m}$. The dashed lines are the suggested high-and low-excitation envelopes. The arrow indicates the effect of reddening. 
lines, and that 6 of the 9 objects with observed HeII $\lambda 4686$ lie near the high-excitation envelope.

It is somewhat puzzling to find that most of the objects with low luminosity $\left(R_{\mathrm{n}}>15 \cdot 1\right)$ lie in the preceding part of the LMC, West of $5^{\mathrm{h}} 20^{\mathrm{m}}$. This could possibly be taken as an effect of higher interstellar reddening; an additional average absorption of $A_{\mathrm{R}}=0.6$ mag West of $5^{\mathrm{h}} 20^{\mathrm{m}}$ is sufficient. However, there is no support for this in observations of other objects, and the observed Balmer decrement of the planetaries is the same; the mean value of $\log F(\mathrm{H} \beta) / F(\mathrm{H} \gamma)$ is 0.30 in both parts of the LMC. A confirmation of the observed difference in $R_{\mathrm{n}}$ is found in the photoelectric flux measures; on the average $\log F(\mathrm{H} \beta)$ is 0.11 fainter in the preceding half of the LMC.

The difference between the planetary nebulae East and West of $5^{\mathrm{h}} 20^{\mathrm{m}}$ was initially noted in the $\left(B_{\mathrm{s}}-B_{\mathrm{n}}\right)$ data in the W-S catalogue (Westerlund and Smith, 1964) and at first suspected as due to a systematic effect in the photographic magnitudes. There was no reason to expect that the central stars should contribute more to the total luminosity of the planetary nebulae in the preceding part than in the following. The effect is, however, obvious also in the narrow-band photoelectric colour indices:

$$
\begin{aligned}
& 5 \text { of } 7 \text { objects West of } 5^{\mathrm{h}} 20^{\mathrm{m}} \text { have } m_{4200}-m_{5300}>0, \text { and only } \\
& 2 \text { of } 11 \text { objects East of } 5^{\mathrm{h}} 20^{\mathrm{m}} \quad m_{4200}-m_{5300}>0 .
\end{aligned}
$$

Furthermore, Webster (1966) has determined the apparent magnitude $m_{\mathrm{s}}$ (4861) of the central star by subtracting the nebular continuum from the 'star plus continuum' observation using its theoretical values. The average corrections are

$$
\begin{aligned}
& m_{\mathrm{s}}(4861)-m_{4200}=+0.4 \text { West of } 5^{\mathrm{h}} 20^{\mathrm{m}} \\
&+0.7 \text { East of } 5^{\mathrm{h}} 20^{\mathrm{m}} .
\end{aligned}
$$

It then appears necessary to accept the results as indicating that there is a systematic difference between the 'average planetary nebula' in the two parts of the LMC.

\section{The Masses of the Planetary Nebulae}

It is well known that the mass of ionized hydrogen in a planetary nebula can be determined provided the distance, angular size, $\mathrm{H} \beta$ flux, electron temperature, and filling factor are known (Minkowski, 1965). For the planetary nebulae in the Magellanic Clouds, the distances are known, the fluxes can be observed, and reasonable assumptions may be made for the electron temperature $\left(T_{\mathrm{e}}=1.5 \times 10^{4}{ }^{\circ} \mathrm{K}\right)$ and the filling factor $(\varepsilon)$. All the objects observed so far are stellar; this gives an upper limit to their radii of about 1 " of arc. Using this value, Webster found the maximum possible mass for the most luminous planetary in the LMC, P 25, to be $1.41 M_{\odot}$ for $\varepsilon=1$. This value is an overestimate, since the radius of $\mathbf{P} 25$ is more likely to be of the order of 0.15 parsec ( $\left(0^{\prime \prime} .5\right)$ from the position of its nucleus in the H-R diagram (Figure 3). Its ionized hydrogen mass is then of the order of $0.45 M_{\odot}$. 
We have determined the masses of two more planetary nebulae selected because of the position of their nuclei in the H-R diagram. The nucleus of the planetary N 6 in the SMC lies near the evolutionary track at $\log T_{\mathrm{s}}=4 \cdot 6$. Its radius is probably smaller than 0.06 parsec. Using this value and the assumptions above, we derive a mass of $0.08 M_{\odot}$. This is low and may indicate that part of the mass of $\mathrm{N} 6$ is not ionized, hence that the object is still optically thick in HI. The planetary $\mathrm{N} 43$ in the SMC is optically thin in $\mathrm{HI}$ and HeI (Webster, 1966). Its central star is faint and its $\mathrm{H} \beta$ flux is among the strongest observed in the Cloud planetaries. We may assume that is has just reached the minimum size for optically thin nebulae, and its radius is then near 0.06 parsec. With $\varepsilon=1$, its hydrogen mass is found to be $0.12 M_{\odot}$.

Webster has applied another method for determining the most likely mass of a planetary nebula, provided there is a unique value. She assumes that the most luminous galactic planetary nebulae are identical to those in the Magellanic Clouds. They are then optically thin in hydrogen and their total emission in $\mathrm{H} \beta$ is $\log E(\mathrm{H} \beta)=35 \cdot 3$. The observed $\mathrm{H} \beta$ flux permits a determination of the distance, and, consequently, of the mass of the galactic object. The true mass will be equal to the lowest mass obtained in this way for the optically thin objects. From calculations for 43 galactic planetary nebulae the best value is found to be $0 \cdot 11 \mathrm{M}_{\odot}$.

We conclude that an average planetary nebula has a hydrogen mass of about $0 \cdot 12$ solar masses, but that masses up to about 0.5 solar masses may be expected.

\section{The Evolution of the Planetary Nebulae}

Webster has determined the temperatures and the luminosities of the central stars of the planetary nebulae in the Magellanic Clouds following Seaton's method. The result is given in Figure 3. The evolutionary track for a distance scale corresponding to a hydrogen mass of $0 \cdot 1 M_{\odot}$ is drawn, as well as Seaton's (dashed) for a hydrogen mass of $0.4 M_{\odot}$. The differences on the high-temperature side are due to the scaling factors only. On the low-temperature side, the $0 \cdot 1 M_{\odot}$ curve is based on improved observations also of the galactic objects.

The high luminosity of the high-temperature nuclei in the Clouds is real. These objects, as well as the low-excitation nebulae in the SMC, have probably slightly higher masses (in the nuclei and in the shell?) than the average nebula. They should not be considered indicating the mean position of the evolutionary track at these temperatures. We note that in Seaton's diagram (1966, Figure 7) a similar situation exists for a group of nuclei at $\log T \geq 5 \cdot 1$. It may be argued, and generally is, that the scatter in our Figure 3 and Seaton's Figure 7 simply indicates the scatter to be expected around one single average mass. However, if the theory is accepted that planetary nebulae evolve from red-giant stars, we have to bear in mind that each portion of the red-giant region in the $\mathrm{H}-\mathrm{R}$ diagram is populated by stars which have evolved from two different luminosity (mass) ranges on the zero-age main sequence. It is then not 
unreasonable to expect to find planetary nebulae of at least two different mass groups.

\section{Conclusions}

The distribution of the planetary nebulae in the Small Magellanic Cloud does not contradict Hindman's model of an edge-on galaxy. The line of symmetry of the planetaries does not, however, agree with the position of the major axis in the HI model. The radial velocities of the planetary nebulae do not indicate that the SMC is a rotating system.

The distribution of the planetary nebulae in the Large Magellanic Cloud is in agreement with a system being seen nearly face-on. The system is rotating, and Webster's analysis indicates that the best centre of rotation is the centroid of the planetary nebulae system.

A number of low-excitation nebulae have been found in the SMC; their character-

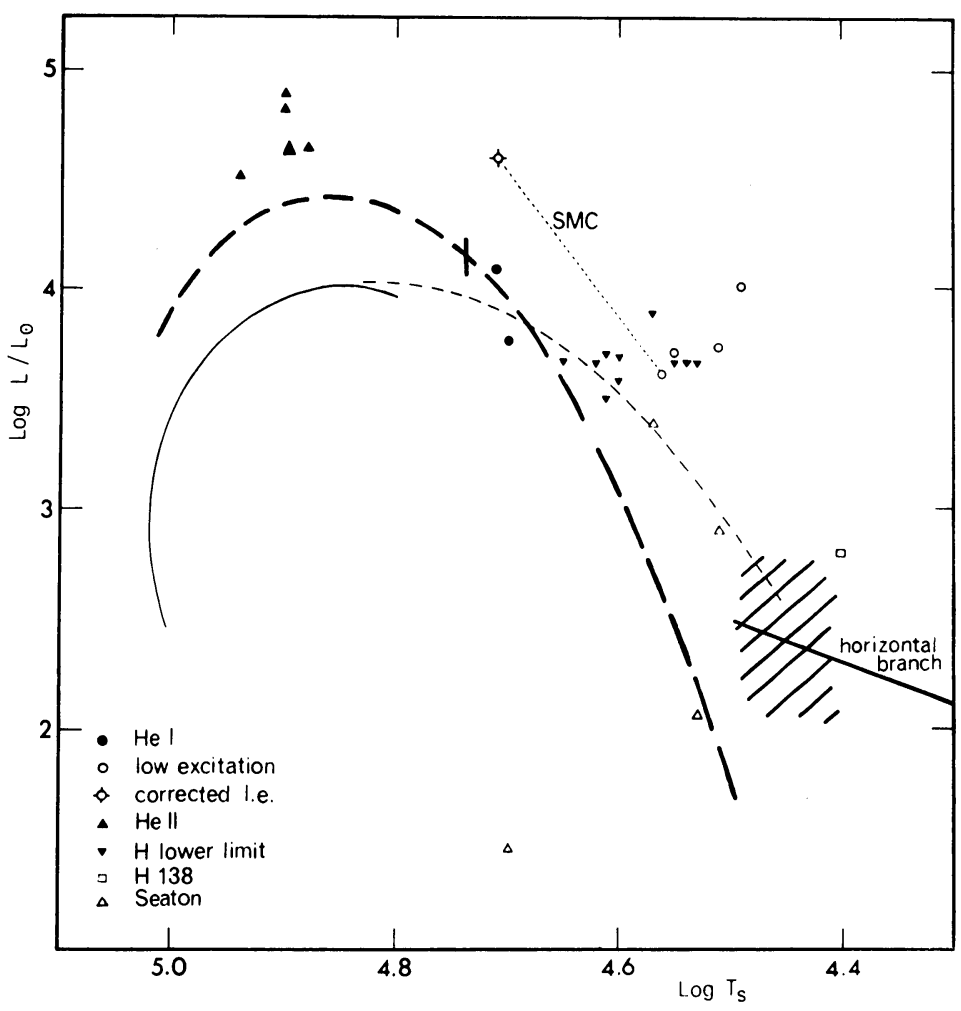

FIG. 3. The evolutionary track of the planetary nuclei according to Webster. The heavy dashes mark Seaton's evolutionary track for a hydrogen mass of $0.4 M_{\odot}$. The vertical line crossing this curve identifies a nebular radius of 0.06 parsec. 
istics are best understood if they have a higher than average mass. Similarly, a group of high-excitation planetary nebulae in the LMC may have masses slightly higher than the average. The best value for the average hydrogen mass of a planetary nebula is found to be near $0 \cdot 1 M_{\odot}$.

In the LMC, we have noted that the planetary nebulae to the West of $5^{\mathrm{h}} 20^{\mathrm{m}}$ are on the average less luminous than those in the Eastern part. At the same time, their nuclei contribute more to their total luminosity. It is unlikely that this is an effect of interstellar reddening or an effect of different distances. A tilt of the LMC of $45^{\circ}$ would cause a differential magnitude effect of $0.04 \mathrm{mag} / \mathrm{kpc}$ (= per degree). This could explain the lower luminosity to some extent, but not the different colors nor the different contribution of the central star to the total luminosity of the planetary nebulae.

\section{References}

Baade, W. (1955) Astr. J., 60, 151.

Cannon, A.J. (1933) Harvard Coll. Obs. Bull., 891.

Feast, M.W. (1964) Observatory, 84, 266.

Feast, M.W., Thackeray, A.D., Wesselink, A.J. (1961) Mon. Not. R. astr. Soc., 122, 433.

Henize, K.G. (1956) Astrophys. J. Suppl. Ser., $2,315$.

Henize, K. G., Westerlund, B.E. (1963) Astrophys. J., 137, 747.

Hindman, J.W. (1967) Austr. J. Phys., 20, 147.

Koelbloed, D. (1956) Observatory, 76, 894.

Lindsay, E. M. (1955) Mon. Not. R. astr. Soc., 115, 248.

Lindsay, E. M. (1956) Mon. Not. R. astr. Soc., 116, 649.

Lindsay, E. M. (1961) Astr. J., 66, 169.

Lindsay, E. M., Mullan, D. J. (1963) Irish astr. J., 6, 51.

McGee, R.X., Milton, J.A. (1966) Austr. J. Phys., 19, 343.

Minkowski, R. (1965) in Galactic Structure, Ed. by A. Blaauw and M. Schmidt, Univ. of Chicago Press, Chicago, p. 321.

O'Dell, C. R. (1962) Astrophys. J., 135, 371.

Seaton, M.J. (1966) Mon. Not. R. astr. Soc., 132, 113.

Śvestka, Z. (1962) Bull. astr. Inst. Csl., 13, 35.

Swope, H. H. (1963) Astr. J., 68, 470.

Webster, B.L. (1965) in Symposium on the Magellanic Clouds, Ed. by J.V. Hindman and B.E. Westerlund, Canberra, p. 29.

Webster, B.L. (1966) Thesis, Austr. Nat. Univ. Canberra.

Westerlund, B. E. (1965) in Symposium on the Magellanic Clouds, Ed. by J. V. Hindman and B.E. Westerlund, Canberra, p. 40.

Westerlund, B.E. (1968) Vistas in Astronomy (in press).

Westerlund, B.E., Rodgers, A.W. (1959) Observatory, 79, 132.

Westerlund, B. E., Smith, L. F. (1964) Mon. Not. R. astr. Soc., 127, 449. 